\title{
Error Estimation on Wander Frame for SINS Ground Alignment Through Observability Analysis
}

\author{
Hadia M. S. EL- Hennawy*, Ibrahim I. Arafa ${ }^{\dagger}$, Alaa M. Fekry*
}

\begin{abstract}
This paper presents a novel alignment method based on wander frame for SINS (strap down inertial navigation system) whose initial attitude information is completely unknown. During the course mode, the cosine and sine of the wander angle is defined as the filter states, and modeled as the velocity/position error in the geographic frame. Azimuth error state in the fine mode of the filter is defined as wander angle error to provide continuous estimation of navigation states. A systematic analysis of the Observability of a strap down inertial navigation system (SINS) in ground alignment with Bar-Itzhack and Berman's error model is presented. It is shown that the unobservable states are separately contained in two de-coupled subspaces. The constraints on the selection of unobservable states are discussed. An estimation algorithm, which is derived fully from the horizontal velocity outputs for computing the misalignment angles, is provided. It reveals that the azimuth error can be entirely estimated from the estimates of leveling error and leveling error rate, without using gyro output signals explicitly. This paper also, estimates the errors of a strap down inertial navigation systems by using appropriate Kalman filter design.
\end{abstract}

Keywords: Inertial Navigation Systems and Kalman Filtering, Inertial Guidance Systems

\section{Nomenclature}

A ellipsoid semi major axis

$A$ azimuth angle

$\mathbf{a}_{\mathrm{s}}$ Vector of measured specific acceleration

B ellipsoid semi minor axis

E Earth eccentricity

F flattening of the ellipsoid

$\mathrm{f}_{\mathrm{E}}, \mathrm{f}_{\mathrm{N}}, \mathrm{f}_{\mathrm{U}}$ East, north, and up specific forces sensed by INS.

$\mathbf{g}_{\mathrm{s}}$ Vector projections in the fixed CS are $[0,0,-\mathrm{g}]$

$R_{E}$ transverse radius of earth curvature

$R_{N}$ meridian radius of earth curvature

$\mathbf{V}_{\mathrm{s}}^{\mathrm{r}}$ Vector of relative velocity in the reference CS $\gamma \quad$ Missile pitch angle

$\Delta_{\mathrm{i}}$ Angular velocity of the earth rate $15.040 / \mathrm{h}=7.292115 \cdot 10^{-5} \mathrm{r} / \mathrm{s}$

$\varepsilon \quad$ Gyro constant drift

$\vartheta \quad$ Missile pitch angle

$\lambda$ Longitudinal angle

$\psi \quad$ Missile pitch angle

$\varphi \quad$ Latitude angle in the place

$\omega_{\mathrm{s}}^{\mathbf{r}}$ Vector of relative rate of a reference CS

$\nabla \quad$ Accelerometer bias

Ain Shams University, Cairo, Egypt.

Egyptian Armed Forces, Egypt.

* Egyptian Armed Forces, Egypt; ala28444@gmail.com 


\section{Introduction}

Initial alignment as the key technique of inertial navigation, can provide the initial value for SINS. Traditionally the processes of initial alignment usually go through two modes after being powered up: the coarse alignment and fine alignment[1]. During the course mode, SINS employ the principle of gyrocompass or earth rate sensing for alignment, given only initial position. The coarse alignment mode determines attitude to within a few degrees. Kalman filter is the common scheme of the fine alignment for today practically all straps down system because of the high estimated accuracy. However, for initial attitude and position uncertainty, above approach is not adapted well.[1].

For Kalman filter application[2], the azimuth angle is predetermined to within a few degrees of accuracy using an unspecified coarse alignment mode, permitting the use of small angle approximations in the error propagation equations of a linearized Kalman filter. But in the actual application, the initial attitude information may be unknown beforehand, or in the failure mode, navigation parameters are destroyed by system sudden interrupt. The traditional Kalman filter model is subjected the new challenge.

The unknown initial attitude is typically handled by modeling the heading error with two states. One design represents the heading error with two error states sin $\emptyset$ err and (1-cos $\emptyset e r r)$. Another method models the level earth rate components $\Omega x, \Omega y$ as Kalman states. However, a problem remains with both methods; if the vehicle is in motion during coarse alignment, position cannot be updated because the wander angle is not sufficiently known to transform wander frame velocity to an earth-fixed frame for integration.

In addition, the Kalman filter cannot accept position fixes as measurement updates in coarse align mode. The organization of this paper proceeds as follows. The next section outlines the design scheme of alignment technique. Section 3 provides the system model based on the wander frame with attitude, velocity, and position error model. In Section 4, onboard mathematical software tasks. In Section 5, IFA error model through Kalman filter mechanization. Numerical simulations and analyses of SINS in-motion alignment are given. Finally, conclusions are drawn.

\section{Alignment Techniques}

This section describes techniques for initialization of inertial navigation system (INS) which is hosted on a missile. The INS initialization aims to determine initial values of the system, including position, velocity, and attitude. In case of alignment on stationary base, it is required to align an inertial navigation system to the local geographic co-ordinate frame defined by the directions of true north and the local vertical [15]. For this purpose, it is assumed that the navigation system is stationary w.r.t. the Earth. That is, the accelerometers measure three orthogonal components of the specific force needed to overcome gravity whilst the gyroscopes measure the components of the Earth's turn rate in the same directions. In a strap down system, attitude information may be stored as a direction cosine matrix. The objective of the angular alignment process is to determine the direction cosine matrix which defines the relationship between the inertial sensor axes and the local geographic frame. The measurements provided by the inertial sensors in body axes may be resolved into the local geographic frame using the current best estimate of the body attitude with respect to this frame. 


\subsection{Analytical INS System}

In this mechanization, the IMU is mounted directly on the host vehicle so that the transformation from the sensor axes to the inertial frame is computed. Therefore, for all practical purposes, in a strap down system the platform frame coincides with the body frame. Consequently, the accelerometers rotate (with respect to inertial space) in the same way as the vehicle. This system is illustrated by the block diagram as shown in figure. (1)[4].

Many frames are involved in Inertial Navigation System (INS) development and analysis because a vector represented in one frame must be transformed into another. The choice of a coordinate system for a specific mechanization depends on many considerations. There are generally five fundamental coordinate frames of interest for expressing motion relative to some frame of reference: (1) Inertial frame, (2) Earth centered fixed frame, (3) Navigation frame, (5) Wander azimuth frame, and (6) Body frame[4]. The inertial frame is not a practical reference frame; it is used only for visualization of other reference frames. This frame is illustrated as shown in figure. (2).

\subsection{Wander Frame Definition}

The wander frame is the same as the local-level frame in all aspects except that its $\mathrm{Y}$ axis is not slaved to the north direction. It is, therefore, allowed to wander off the North axis at a rate chosen by the user. The angle between the Y and North axes is called the wander angle. The relationship between the local level frame and wander frame is shown on Fig.2.

According to the introduction of wander angle $\alpha$, the equation of $\dot{\alpha}$ is expressed:

$$
\dot{\alpha}=-\frac{\mathrm{v}_{\mathrm{x}}}{\mathrm{R}} \operatorname{tg} \varphi
$$

where $\mathrm{Vx}$ is the east velocity in local-level frame, $\varphi$ is the latitude and $\mathrm{R}$ is the radius of the earth.

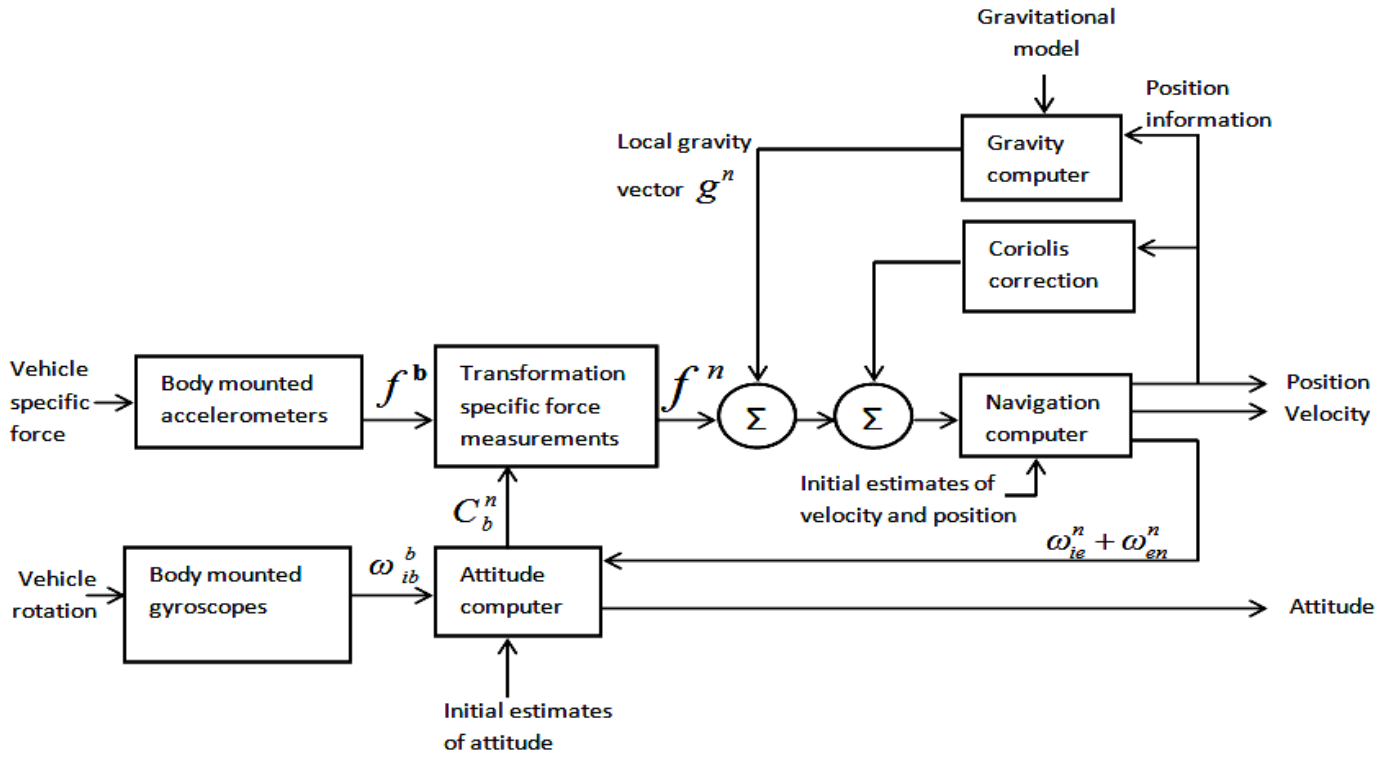

Fig.1. Strap down (Analytical) INS System 


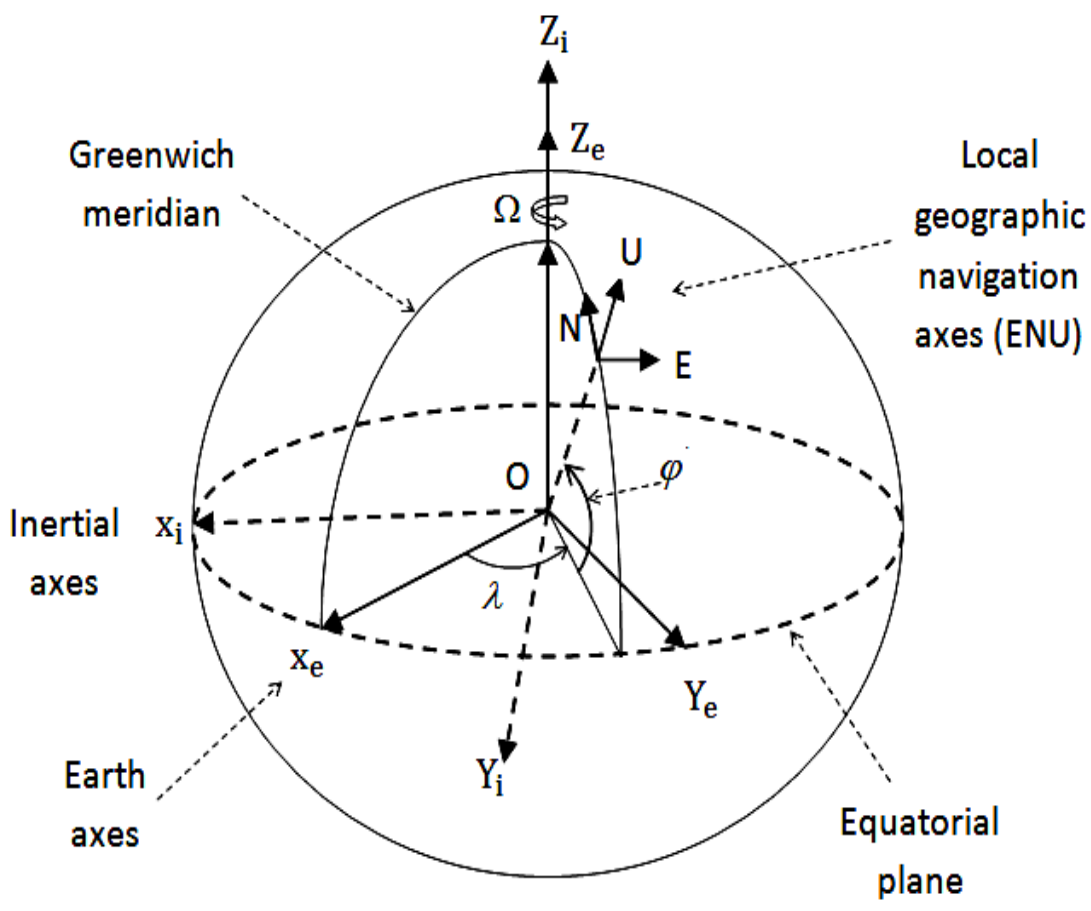

Fig.2.INS Coordinate frames

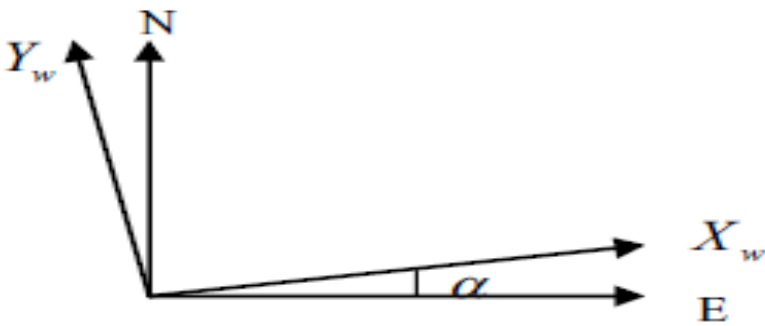

Fig.3. Relationship between the local-level frame and wander frame

The definition of the wander frame is the following: origin is at top center; $\mathrm{X}_{\mathrm{W}}$ axis is rotated in the level plane by an angle $\alpha$ from the east toward the north. The $Y_{w}$ axis is orthogonal to the $\mathrm{X}$-axis in the level plane; $\mathrm{Z}_{\mathrm{W}}$ axis is upwards along the ellipsoidal normal.

\subsection{Attitude Error Model}

The mechanization equations for a local level INS using a wander azimuth navigation frame are as follows.

Attitude with respect to the wander frame is determined from a direction cosine $C_{b}^{w}$ which is updated with

$$
\begin{gathered}
\dot{\mathrm{C}}_{\mathrm{b}}^{\mathrm{w}}=\mathrm{C}_{\mathrm{b}}^{\mathrm{w}}\left[\omega_{\mathrm{ib}}^{\mathrm{b}} \mathrm{x}\right]-\left[\omega_{\mathrm{iw}}^{\mathrm{w}} \mathrm{x}\right] \mathrm{C}_{\mathrm{b}}^{\mathrm{w}} \\
\omega_{\mathrm{iw}}^{\mathrm{w}}=\omega_{\mathrm{ie}}^{\mathrm{w}}+\omega_{\mathrm{ew}}^{\mathrm{w}}
\end{gathered}
$$

where, $\omega_{\mathrm{ib}}^{\mathrm{b}}$ is spatial rate of vehicle, in body frame as measured by gyro; $\omega_{\mathrm{iw}}^{\mathrm{w}}$ is spatial rate of wander frame, expressed in wander frame; $\omega_{\mathrm{ie}}^{\mathrm{W}}$ is spatial rate of the earth, expressed in wander frame; $\omega_{\mathrm{ew}}^{\mathrm{W}}$ is transport rate, or rotational rate of wander frame with respect to the earth, expressed in wander frame. 


$$
\begin{gathered}
\omega_{\mathrm{ie}}^{\mathrm{w}}=\Omega\left\{\begin{array}{lll}
\cos \mathrm{L} \sin \alpha & \cos \mathrm{L} \cos \alpha & \sin \mathrm{L}
\end{array}\right\} \\
\omega_{e w}^{W}=\left\{\begin{array}{lll}
\rho_{x} & \rho_{y} & 0
\end{array}\right\}
\end{gathered}
$$

And the cross-product form $[\omega \mathrm{x}]$ of a vector is skew - symmetric matrix:

$$
[\omega x]=\left[\begin{array}{ccc}
0 & -\omega_{z} & \omega_{y} \\
\omega_{z} & 0 & \omega_{x} \\
-\omega_{y} & -\omega_{x} & 0
\end{array}\right]
$$

The velocity in wander frame $\mathrm{V}^{\mathrm{w}}$ is updated with

$$
\dot{\mathrm{V}}_{\mathrm{W}}=\mathrm{C}_{\mathrm{b}}^{\mathrm{w}} \mathrm{f}^{\mathrm{b}}-\left(2 \omega_{\mathrm{ie}}^{\mathrm{w}}+\omega_{\mathrm{ew}}^{\mathrm{w}}\right) * \mathrm{~V}^{\mathrm{w}}+\mathrm{g}^{\mathrm{w}}
$$

where $\mathrm{f}^{\mathrm{b}}$ body axis specific force is sensed by strapdown accelerometer; $\mathrm{g}^{\mathrm{w}}$ is gravity in wander frame.

Geographic position is given by the direction cosine $\mathrm{C}_{\mathrm{e}}^{\mathrm{W}}$ relating the wander frame to the earth-fixed frame:

$$
\dot{\mathrm{C}}_{\mathrm{W}}^{\mathrm{e}}=\mathrm{C}_{\mathrm{w}}^{\mathrm{e}}\left[\omega_{\mathrm{ew}}^{\mathrm{W}} \mathrm{X}\right]
$$

For a mechanization using a wander frame that is oriented at an azimuth angle $\alpha$ from the East - north - up frame, the level component $\rho_{\mathrm{x}}, \rho_{\mathrm{y}}$ of the transport rate $\omega_{\mathrm{ew}}^{\mathrm{W}}$ are computed from the level components $V_{x}, V_{y}$ of wander velocity $V^{w}$ with

$$
\begin{aligned}
& \rho_{x}=V_{y}\left(\frac{C^{2} \alpha}{R_{n}}+\frac{S^{2} \alpha}{R_{m}}\right)+V_{x} C \alpha S \alpha\left(\frac{1}{R_{n}}-\frac{1}{R_{m}}\right) \\
& \rho_{y}=V_{x}\left(\frac{C^{2} \alpha}{R_{m}}+\frac{S^{2} \alpha}{R_{n}}\right)+V_{y} C \alpha S \alpha\left(\frac{1}{R_{m}}-\frac{1}{R_{n}}\right)
\end{aligned}
$$

where the meridional radius of curvature $R_{m}$ and the principal normal radius $R_{n}$ are computed from the earth ellipticity $\mathrm{e}^{2}$, major axis a and geodetic latitude $\mathrm{L}$

$$
\begin{gathered}
\mathrm{R}_{\mathrm{n}}=\mathrm{a} /\left(1-\mathrm{e}^{2} \sin ^{2} \mathrm{~L}\right)^{\frac{1}{2}} \\
\mathrm{R}_{\mathrm{m}}=\mathrm{a}\left(1-\mathrm{e}^{2}\right) /\left(1-\mathrm{e}^{2} \sin ^{2} \mathrm{~L}\right)^{\frac{3}{2}}
\end{gathered}
$$

The position is updated with Eq.13 and Eq.14, using the estimate $\mathrm{C} \alpha$ and $\mathrm{S} \alpha$

$$
\begin{gathered}
\dot{L}=\frac{1}{R_{m}}\left(V_{x} C \alpha-V_{y} S \alpha\right) \\
\dot{\lambda}=\frac{1}{R_{n} \operatorname{Cos} \alpha}\left(V_{x} S \alpha-V_{y} C \alpha\right)
\end{gathered}
$$

where $\mathrm{C} \alpha, \mathrm{S} \alpha$ are the estimates of sine and cosine of wander angle. Normally, the initial values of $C \alpha$ and $S \alpha$ are zero.

In coarse alignment mode, the wander angle error $\delta \alpha$ probably is very large. The rational for choosing sine and cosine of wander angle $(\delta S \alpha, \delta C \alpha)$ as states is that the error equations, though non-linear in heading error, are linear in $\mathrm{S} \alpha$ and $\mathrm{C} \alpha$, permitting the design of an extended Kalman filter.

Eq. 15 describes the error propagation for the filter model in coarse mode. 


\section{$X=\left[\begin{array}{llllllllll}\delta L & \delta \lambda & \delta h & \delta V_{x} & \delta V_{y} & \delta V_{z} & \phi_{x} & \phi_{y} & \delta c \alpha & \delta s \alpha\end{array}\right]$}

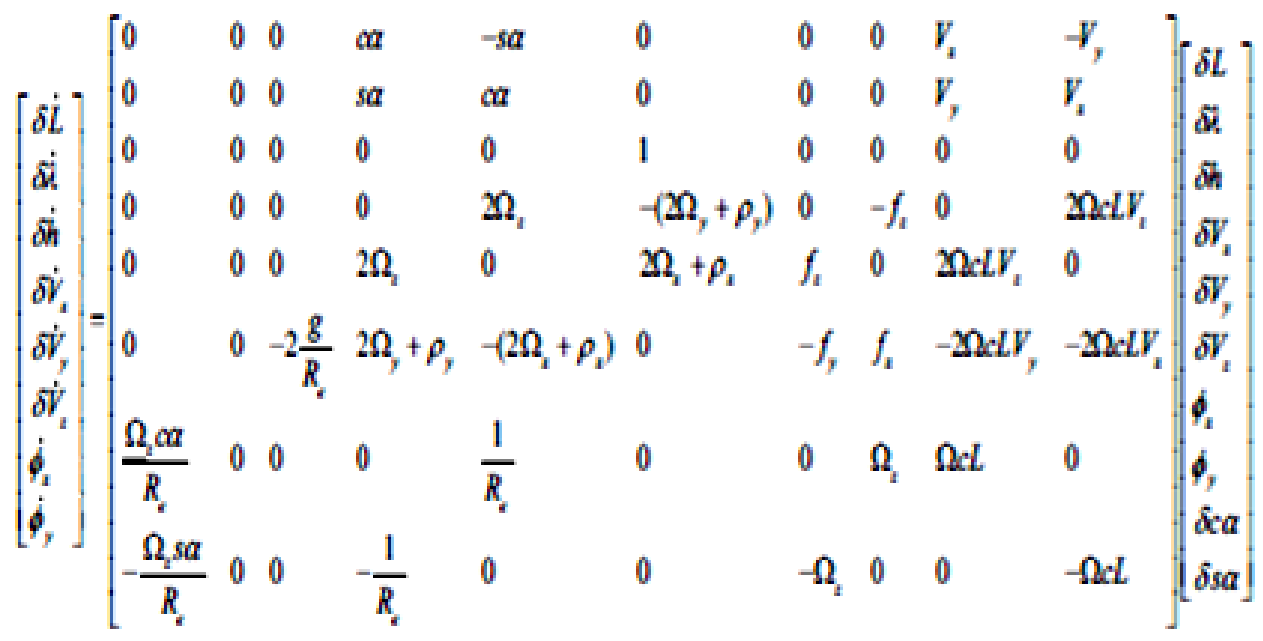

Doppler measured body frame velocity is transformed to wander frame, then subtracted from the inertial velocity. The observation matrix for the velocity difference is obtained from [3]

$$
\begin{aligned}
\Delta \mathrm{V}^{\mathrm{w}} & =\mathrm{V}_{\mathrm{INS}}^{\mathrm{w}}-\mathrm{C}_{\mathrm{b}}^{\mathrm{W}} \mathrm{V}_{\mathrm{DVL}}^{\mathrm{b}} \\
& =\delta \mathrm{V}_{\mathrm{INS}}^{\mathrm{w}}-\left[\delta \mathrm{C}_{\mathrm{b}}^{\mathrm{W}}\right] \mathrm{V}^{\mathrm{b}}-\mathrm{C}_{\mathrm{b}}^{\mathrm{w}} \delta \mathrm{V}_{\mathrm{DVL}}^{\mathrm{b}} \\
& =\left[\begin{array}{l}
\delta \mathrm{V}_{\mathrm{x}} \\
\delta \mathrm{V}_{\mathrm{y}} \\
\delta \mathrm{V}_{\mathrm{z}}
\end{array}\right]-\left[\begin{array}{cr}
0 & -\mathrm{V}_{\mathrm{z}} \\
\mathrm{V}_{\mathrm{z}} & 0 \\
-\mathrm{V}_{\mathrm{y}} & \mathrm{V}_{\mathrm{x}}
\end{array}\right]\left[\begin{array}{l}
\phi_{\mathrm{x}} \\
\phi_{\mathrm{y}}
\end{array}\right]-\mathrm{C}_{\mathrm{b}}^{\mathrm{w}} \delta \mathrm{V}_{\mathrm{DVL}}^{\mathrm{b}}
\end{aligned}
$$

The Doppler error $\delta \mathrm{V}_{\mathrm{DVL}}^{\mathrm{b}}$ may be expanded into biases, scale factor errors, and nonorthogonalities, depending on the application.

In the fine alignment mode, the two states $\delta \mathrm{C} \alpha$ and $\delta S \alpha$ are replaced by a single azimuth error state $\delta \alpha$. The error equation of fine mode is shown in eq .17 where azimuth error is model as error in $\delta \alpha$. As the same as the coarse alignment error equation, the inertial instrument errors is added to model depend on the application.

\subsection{On-Board Computer Navigation Software}

The On-Board Computer Navigation Software (OBCNS) is a complex system with many functions. It performs the following functions as shown in figure (5): interface with ground support equipment, the majority of system test and checkout, ground processing, launch and all of the navigation, guidance, and control and sequencing functions required for flight.

The OBS development comprised of 3 major components: 1) the operating system, IMU digital signal processing code and ground alignment software and 2) ground checkout code developed uniquely for the program system and 3) the flight guidance, navigation, control and sequencing code. 


$$
\begin{aligned}
& X=\left[\begin{array}{lllllllll}
\delta L & \delta \lambda & \delta h & \delta V_{x} & \delta V_{y} & \delta V_{z} & \phi_{x} & \phi_{y} & \delta \alpha
\end{array}\right]
\end{aligned}
$$

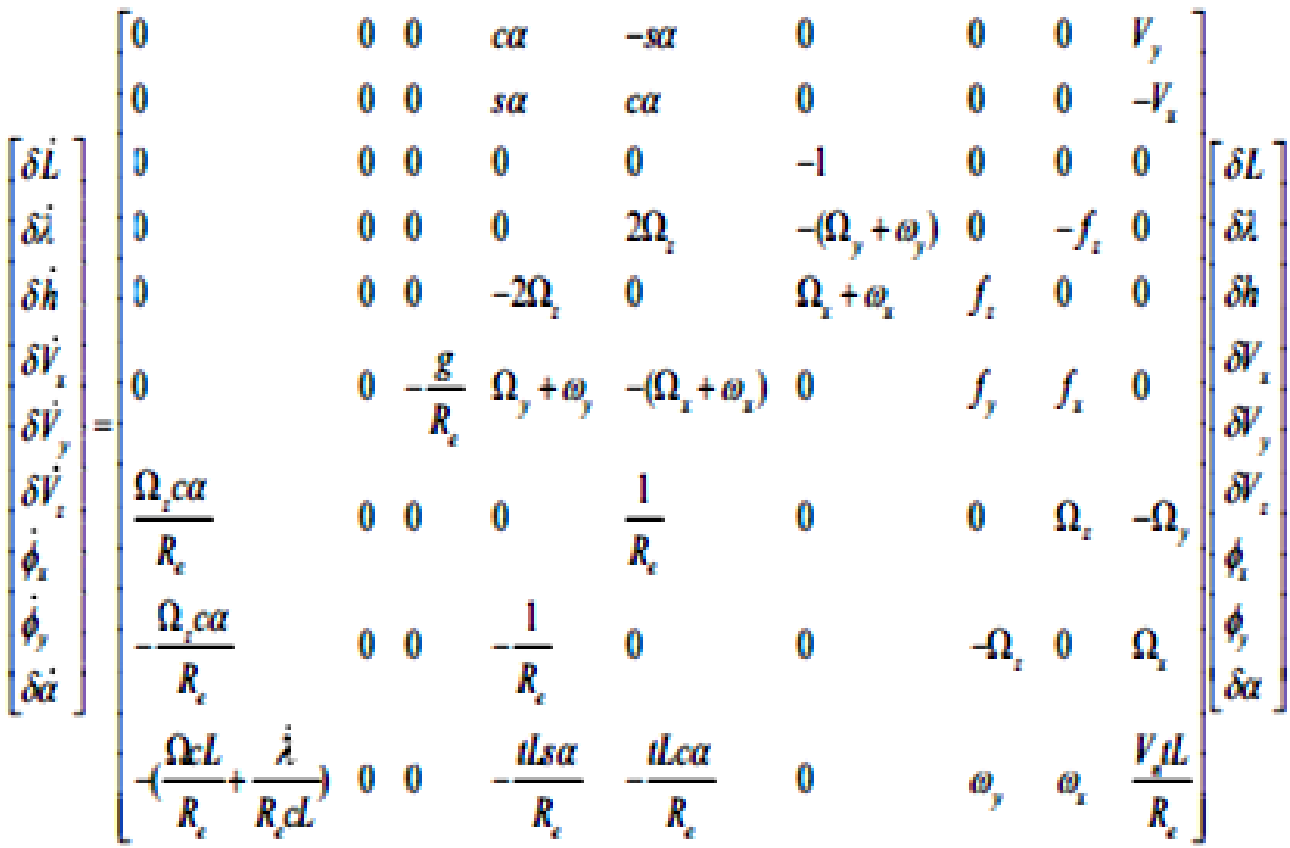

The flight navigation routines are dependent on inputs from the IMU sensors (gyros, accelerometers) [6]. The compensated sensor outputs; Incremental angles and velocities are accumulated and made available at 256 [Hz].Additional compensations are also implemented in the flight inertial navigation algorithm to compensate for the estimated instrument parameters via the System Error Estimator (SEE) updates [6,7]. The SEE is a fifteen states Kalman filter algorithm that utilizes the position error, as 3 scalar measurements, between the propagated inertial position and GPS receiver solution.

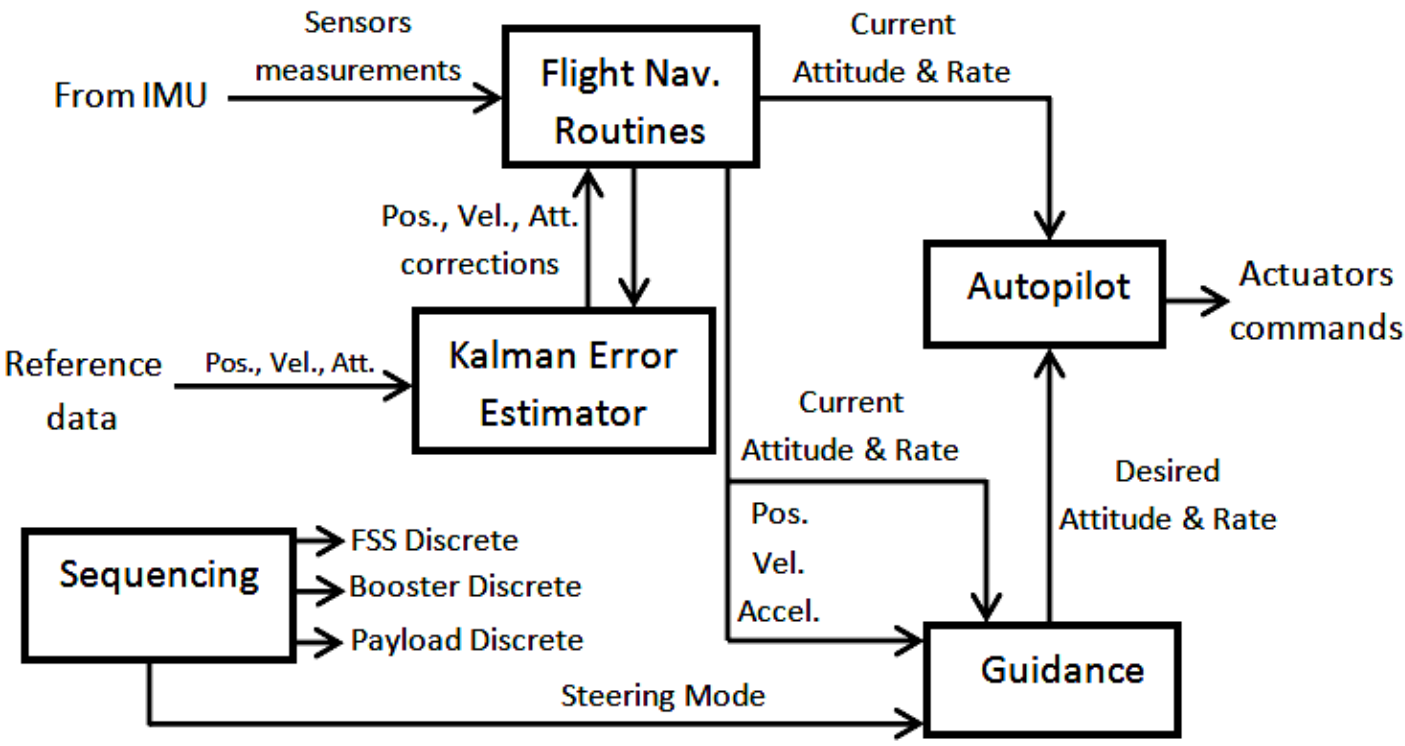

Fig.4. Onboard computer flight functional diagram

After the final implementation integrates the guidance kit with the flight navigation solution via the Kalman filter: There are two modes of operation for the system: 1) ground test which assure the navigation system is healthy before launch and 2) navigation in flight. 
In the ground test we compute the system azimuth, level orientation and auxiliary sensor compensation through the ground alignment time. This creates the initial attitude reference and gyro and accelerometer biases and scale factors which are used at the time of (Go Inertial) for the flight navigation system $[7,8]$.

\subsection{Protocol of Mathematical Tasks}

Here we describe the function of an operating program, functional modules and interrupt handlers as part of the Navigation software.

The Navigation mathematical software (NMSW) performs the following functions [15]:

- Support for INS initial alignment

- Solution of navigation problems: inertial velocity and coordinate reckoning.

- Interaction with navigation equipment and external users of navigation data.

- Support for inertial sensors calibration.

The interaction with users and external systems is provided using ARINC-429 interface. The NMSW is intended for operation as part of IMU in the central processor. The NMSW is written in $\mathrm{C} / \mathrm{C}++$ high-level language using modular and object-oriented programming methods. Assembler is used for creating time critical modules

\section{Navigation Mathematical Algorithms}

Transformation matrix between Wander azimuth launch coordinate system and body fixed system is given by:

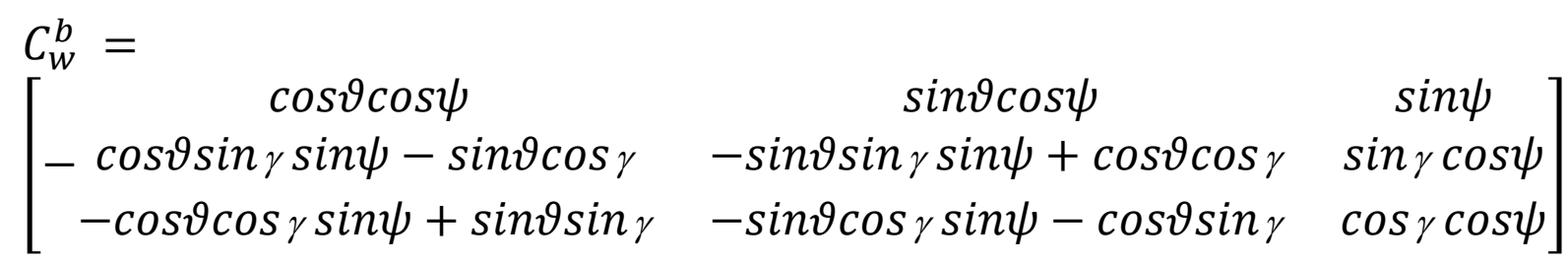

The expressions for the orientation angles $(\vartheta, \gamma, \psi)$ take the form:

$$
\begin{gathered}
\theta=\tan ^{-1}\left(\frac{c_{12}}{c_{11}}\right) \\
\gamma=\tan ^{-1}\left(\frac{c_{23}}{c 33}\right) \\
\psi=\sin ^{-1}\left(c_{13}\right)
\end{gathered}
$$

\subsection{Earth Model}

Ellipsoid of rotation with the axis coinciding with the Earth rotation axis can be used as the Earth navigational model relative to the systems, where the method of inertial navigation is implemented:

$\mathrm{e}=\sqrt{[\mathrm{f}(2-\mathrm{f})]} \quad ; \quad \mathrm{e}^{2}=\frac{\mathrm{a}^{2}-\mathrm{b}^{2}}{\mathrm{a}^{2}} ; \mathrm{f}=\frac{\mathrm{a}-\mathrm{b}}{\mathrm{a}} ;$ 
WGS-84 international reference-ellipsoid with the following parameters is selected to be used in NMSW:

$a=6378137 \mathrm{~m}$

$f=1 / 298.257223563$

$\mathrm{e}^{2}=6.6943799901413 * 10^{-3}[\mathrm{~m}]$

\subsection{Velocity Integration and Navigational Calculations}

To obtain relative velocity in the reference CS, the following differential equation is integrated:

$$
\dot{\mathbf{V}_{\mathrm{s}}^{\mathbf{r}}}=\mathbf{a}_{\mathrm{s}}-\left(\omega_{\mathrm{s}}^{\mathbf{r}}+2 \mathbf{U}_{\mathrm{s}}\right) \times \mathbf{V}_{\mathrm{s}}^{\mathbf{r}}+\mathbf{g}_{\mathrm{s}}
$$

The vector of velocity increment $\delta \mathbf{V}_{\mathrm{S}}$ can be obtained from the processing of accelerometer readings (the compensation of instrument errors). The vector of relative velocity in the reference trihedral takes the form $[11,15]$ :

$$
\begin{aligned}
& \mathbf{V}_{\mathbf{s}}^{\mathbf{r}}=\delta \mathbf{V}_{\mathrm{s}}-\left(\boldsymbol{a}_{s}^{k}-\mathbf{g}_{\mathrm{s}}\right)\left(\mathbf{t}_{\mathbf{m}}-\mathbf{t}_{\mathbf{m}-1}\right) \\
& \boldsymbol{a}_{s}^{k}=\left(\omega_{\mathrm{s}}^{\mathbf{r}}+2 \mathbf{U}_{\mathrm{s}}\right) \times \mathbf{V}_{\mathrm{s}}^{\mathbf{r}}
\end{aligned}
$$

The components of relative rate of reference trihedral on their proper axes have the following form [15]:

$$
\begin{gathered}
\omega_{\mathrm{s} 1}^{\mathbf{r}}=-\mathbf{k}_{\mathrm{s} 2} \mathbf{V}_{\mathrm{s} 2}^{\mathbf{r}}+\mathbf{k}_{\mathrm{s} 3} \mathbf{V}_{\mathrm{s} 1}^{\mathbf{r}} \\
\omega_{\mathrm{s} 2}^{\mathbf{r}}=\mathbf{k}_{\mathrm{s} 1} \mathbf{V}_{\mathrm{s} 1}^{\mathbf{r}}-\mathbf{k}_{\mathrm{s} 3} \mathbf{V}_{\mathrm{s} 2}^{\mathbf{r}} \\
\omega_{\mathrm{s} 3}^{\mathbf{r}}=\mathbf{k}_{\mathbf{E}} \mathbf{V}_{\mathbf{E}} \tan \varphi
\end{gathered}
$$

The curvature of a flight path in the direction of the axes of the reference trihedral is given by:

$$
\begin{gathered}
\mathbf{k}_{\mathrm{s} 1}=\mathbf{k}_{\mathbf{E}} \cos ^{2} \mathbf{A}+\mathbf{k}_{\mathbf{N}} \sin ^{2} \mathbf{A} \\
\mathbf{k}_{\mathrm{s} 2}=\mathbf{k}_{\mathbf{E}} \sin ^{2} \mathbf{A}+\mathbf{k}_{\mathbf{N}} \cos ^{2} \mathbf{A} \\
\mathbf{k}_{\mathrm{s} 3}=\left(\mathbf{k}_{\mathbf{N}}-\mathbf{k}_{\mathbf{E}}\right) \sin \mathbf{A} \cos \mathbf{A} \\
\mathbf{k}_{\mathbf{E}}=\frac{1}{\mathbf{R}_{\mathbf{E}}} ; \mathbf{k}_{\mathbf{N}}=\frac{1}{\mathbf{R}_{\mathbf{N}}} ; \mathbf{k}_{\mathbf{N}}=\mathbf{k}_{\mathbf{E}} \frac{1-\mathbf{e}^{2} \sin ^{2} \varphi}{1-\mathbf{e}^{2}} \\
\mathbf{R}_{\mathbf{N}}=\mathbf{R}_{\mathbf{E}} \frac{1-\mathbf{e}^{2}}{1-\mathbf{e}^{2} \sin ^{2} \varphi} ; \mathbf{R}_{\mathbf{E}} \cong \frac{\mathbf{a}}{\sqrt{1-\mathbf{e}^{2} \sin ^{2} \varphi}}+\mathbf{h}=\mathbf{N}+\mathbf{h}
\end{gathered}
$$

The components of Earth rate along the axes of the reference trihedral can be calculated by $[11,15]$ :

$$
\mathbf{U}_{\mathrm{s} 1}=-\Omega \cos \phi \sin A ; \quad \mathbf{U}_{\mathrm{s} 2}=\Omega \cos \varphi \cos A ; \quad \mathbf{U}_{\mathrm{s} 3}=\Omega \sin \varphi(33)
$$

The absolute angular rate of the reference CS is given by:

$$
\omega_{s}^{a}=\omega_{s}^{r}+U_{s}
$$


The components of a transition velocity of Earth rotation are:

$$
V_{s 1}^{e}=R_{E} U_{s 2} \quad ; V_{s 2}^{e}=-R_{E} U_{s 1} \quad ; \quad V_{s 3}^{e}=0
$$

The vector of absolute velocity in the reference trihedral is given by:

$$
V_{s}^{a}=V_{s}^{r}+V_{s}^{e}
$$

The components of a relative linear velocity in the navigation trihedral are [15]:

$$
\begin{gathered}
V_{E}^{r}=V_{s 1}^{r} \cos A+V_{S 2}^{r} \sin A ; \\
V_{N}^{r}=-V_{s 1}^{r} \sin A+V_{S 2}^{r} \cos A \\
V_{U}^{r}=V_{s 3}^{r}
\end{gathered}
$$

\subsection{Calculation Algorithm of Navigation Coordinates}

Navigation algorithm is in the integration of $\dot{\mathbf{C}}^{\mathbf{N a v}}=\hat{\boldsymbol{\omega}} \times \mathbf{C}^{\text {Nav }}$ Poisson equation and thence finding the matrix of direction cosines of navigation trihedral orientation, and hence geographic coordinates: $\varphi$ (latitude), $\lambda$ (longitude), $A$ (azimuth) based on $\mathrm{C}^{\text {Nav }}(0)=\mathrm{f}\left(\varphi_{0}, \lambda_{0}, \mathrm{~A}_{0}\right)$ initial value and $\omega$ angular rate of a navigation trihedral in the projections on its proper axes that is calculated in " $n$-th" step by the value of relative linear velocity obtained as a result of the integration of accelerometer readings with regard to calculated values of Earth movement parameters, gravitational acceleration, Coriolis acceleration and the radius of curvature of Earth's surface.

Poisson equation is integrated on the basis of vector calculated value of $\boldsymbol{\omega}_{s}^{r}$ relative rate, and the matrix of direction cosines of navigation trihedral can be determined by formulas:

$$
\begin{gathered}
\dot{C}^{N a v}=\hat{\omega}_{s}^{r} \times C^{N a v} \\
\hat{\omega}_{s}^{r}=\left[\begin{array}{ccc}
0 & \omega_{3} & -\omega_{2} \\
-\omega_{3} & 0 & \omega_{1} \\
\omega_{2} & -\omega_{1} & 0
\end{array}\right] \\
C^{\text {Nav }}\left(\mathrm{t}_{0}\right)=\left(\begin{array}{ccc}
\sin \varphi \cdot \cos \lambda \cdot \sin \mathrm{A}-\sin \lambda \cdot \cos \mathrm{A} & \sin \varphi \cdot \sin \lambda \cdot \sin \mathrm{A}+\cos \lambda \cdot \cos \mathrm{A} & -\cos \varphi \cdot \sin \mathrm{A} \\
-\sin \varphi \cdot \cos \lambda \cdot \cos \mathrm{A}-\sin \lambda \cdot \sin \mathrm{A} & \cos \lambda \cdot \sin \mathrm{A}-\sin \varphi \cdot \sin \lambda \cdot \cos \mathrm{A} & \cos \varphi \cdot \cos \mathrm{A} \\
\cos \varphi \cdot \cos \lambda & \cos \varphi \cdot \sin \lambda & \sin \varphi
\end{array}\right) \\
\boldsymbol{A}=\arctan \frac{\arctan \frac{-\mathbf{c}_{13}}{\mathbf{c}_{33}}[-\pi, \pi]}{\mathbf{c}_{31}^{2}+\mathbf{c}_{32}^{2}}\left[-\frac{\pi}{2}, \frac{\pi}{2}\right] ;
\end{gathered}
$$

\subsection{In flight Alignment Error model}

We introduce the error model, which appropriately describes the INS behavior during IFA. We then substantiate its adequacy for consideration as a piece-wise constant system (PWCS) and then the simpler stripped Observability matrix (SOM) can be used in the system 
Observability analysis. The most suitable model for IFA, where the measured states are the velocity states, is the, so called psi-model $[11,12,13,14]$. This model is in the form:

$$
\begin{gathered}
{\left[\begin{array}{l}
\delta \dot{V}_{x} \\
\delta \dot{V}_{y} \\
\delta \dot{V}_{z} \\
\dot{\psi}_{x} \\
\dot{\psi}_{y} \\
\dot{\psi}_{z}
\end{array}\right]=\left[\begin{array}{cccccc}
0 & \tilde{\Omega}_{\mathrm{U}} & -\tilde{\Omega}_{\mathrm{N}} & 0 & -f_{\mathrm{U}} & f_{\mathrm{N}} \\
-\tilde{\Omega}_{\mathrm{U}} & 0 & \tilde{\Omega}_{\mathrm{E}} & f_{\mathrm{U}} & 0 & -f_{\mathrm{E}} \\
\tilde{\Omega}_{\mathrm{N}} & -\tilde{\Omega}_{\mathrm{E}} & 0 & -f_{\mathrm{N}} & f_{\mathrm{E}} & 0 \\
0 & 0 & 0 & 0 & \Omega_{U} & -\Omega_{N} \\
0 & 0 & 0 & -\Omega_{U} & 0 & \Omega_{E} \\
0 & 0 & 0 & \Omega_{N} & -\Omega_{E} & 0
\end{array}\right]\left[\begin{array}{l}
\delta V_{x} \\
\delta V_{y} \\
\delta V_{z} \\
\psi_{x} \\
\psi_{y} \\
\psi_{z}
\end{array}\right]+\left[\begin{array}{c}
\nabla_{x} \\
\nabla_{y} \\
\nabla_{z} \\
\varepsilon_{x} \\
\varepsilon_{y} \\
\varepsilon_{z}
\end{array}\right] \text { (4) }} \\
\tilde{\mathbf{\Omega}}=\left[\begin{array}{ccc}
0 & \tilde{\Omega}_{\mathrm{U}} & -\tilde{\Omega}_{N} \\
-\tilde{\Omega}_{\mathrm{U}} & 0 & \tilde{\Omega}_{E} \\
\tilde{\Omega}_{N} & -\tilde{\Omega}_{E} & 0
\end{array}\right] ; \quad \boldsymbol{\Omega}=\left[\begin{array}{ccc}
0 & \Omega_{U} & -\Omega_{\mathrm{N}} \\
-\Omega_{U} & 0 & \Omega_{E} \\
\Omega_{\mathrm{N}} & -\Omega_{E} & 0
\end{array}\right] ; \mathbf{F}_{j}=\left[\begin{array}{ccc}
0 & -f_{\mathrm{U}} & f_{\mathrm{N}} \\
f_{\mathrm{U}} & 0 & -f_{\mathrm{E}} \\
-f_{\mathrm{N}} & f_{\mathrm{E}} & 0
\end{array}\right]
\end{gathered}
$$

Consequently, the carrier trajectory during the IFA maneuver can be characterized by a sequence of segments taken from table (1). The elements $\mathbf{f}_{\mathbf{E}}, \mathbf{f}_{\mathbf{N}}$, and $\mathbf{f}_{\mathbf{U}}$ for each segment $\mathrm{j}$ are the entries of the sub matrices $F_{j}$

Table 1.Trajectory segmentations of the missile

\begin{tabular}{|c|l|l|l|l|}
\hline No. of Segment (j) & Maneuver Characteristics & $\mathrm{f}_{\mathrm{E}}$ & $\mathrm{f}_{\mathrm{N}}$ & $\mathrm{f}_{\mathrm{U}}$ \\
\hline $\mathbf{1}$ & straight flight & 0 & 0 & $-\mathrm{g}$ \\
\hline $\mathbf{2}$ & maneuver with north acceleration & 0 & $\mathrm{f}_{\mathrm{N}}$ & $-\mathrm{g}$ \\
\hline $\mathbf{3}$ & maneuver with horizontal acceleration & $\mathrm{f}_{\mathrm{E}}$ & $\mathrm{f}_{\mathrm{N}}$ & $-\mathrm{g}$ \\
\hline
\end{tabular}

The Observability analysis of the INS at segment $\mathrm{j}$, after having gone through segments $1,2, \ldots \mathrm{j}-1$ from table (1), the total Observability matrix (TOM) $\widetilde{Q}(\mathrm{j})$ is constructed as follows [14]:

$$
\tilde{Q}(j)=\left[\begin{array}{c}
\tilde{Q}_{1} \\
\tilde{Q}_{2} \mathrm{e}^{A_{1} \Delta_{1}} \\
\vdots \\
\tilde{Q}_{j} \mathrm{e}^{A_{j-1} \Delta j-1} \ldots \mathrm{e}^{A_{1} \Delta_{1}}
\end{array}\right]
$$

where

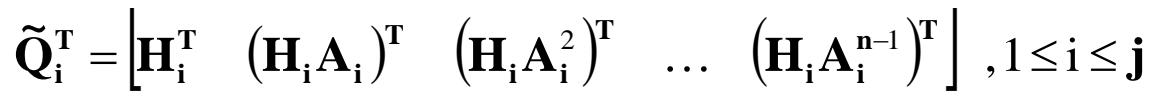

Consequently, instead of using the TOM of Equation (27) we may use $\tilde{Q}_{s}(j)$ stripped Observability matrix (SOM), which simplifies the Observability analysis considerably. The $\operatorname{SOM} \tilde{\mathbf{Q}}_{\mathbf{s}}(\mathbf{j})$ is constructed as[14]: 


$$
\tilde{Q}_{s}(j)=\left[\begin{array}{c}
\tilde{Q}_{1} \\
\tilde{Q}_{2} \\
\vdots \\
\tilde{Q}_{j}
\end{array}\right]
$$

\subsection{Simulation and Results}

This simulation built on the hard ware in the loop (HIL) block diagram which describes the missile real time flight simulation test mode using six-degree of freedom simulator as shown in Figure (5) and table (2) shows the missile Initial parameters.

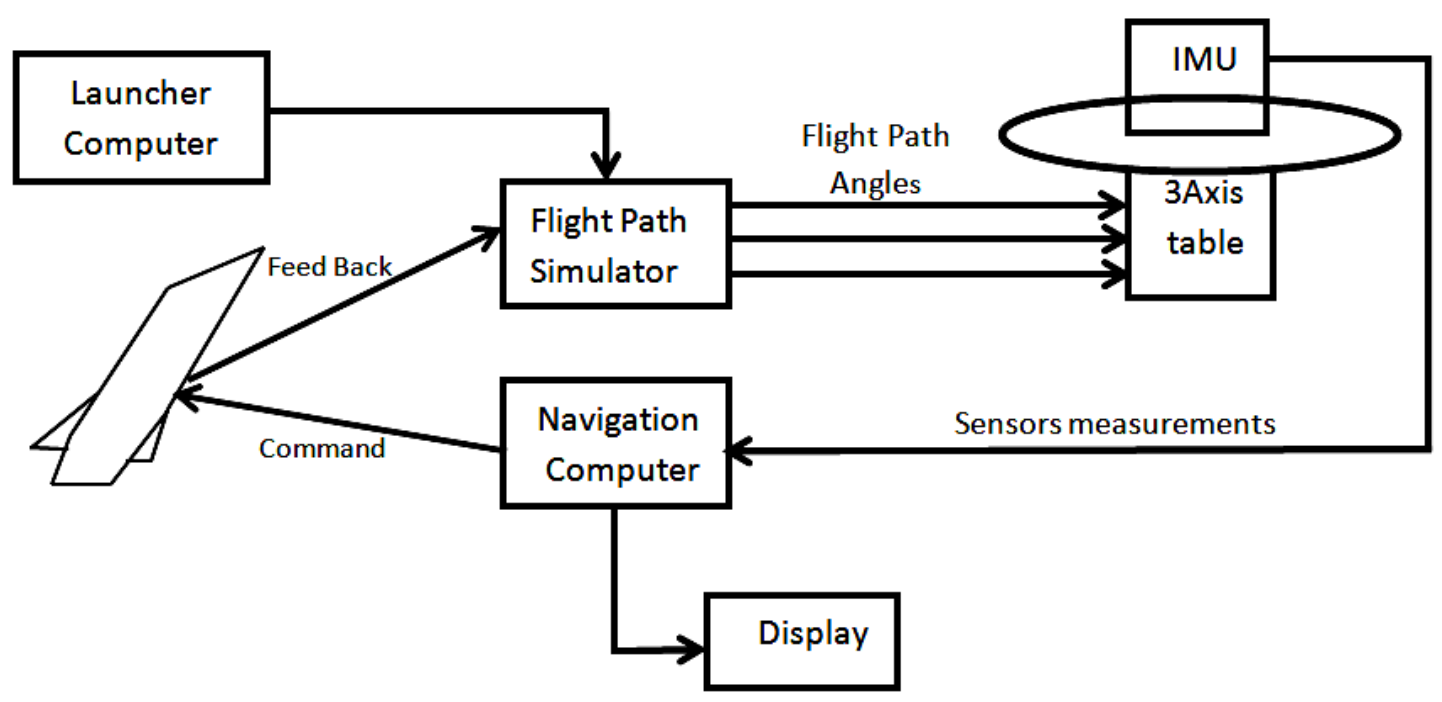

Fig.5. HIL flight simulation test mode.

Table 2. Initial flight parameters of the missile

\begin{tabular}{|l|l|l|l|}
\hline \multicolumn{4}{|c|}{ Trajectory initial parameters } \\
\hline Initial pitch angle & $90.00[\mathrm{deg}]$ & Accelerometer bias & $10[\mu \mathrm{g}]$ \\
\hline Initial latitude angle & $30.1[\mathrm{deg}]$ & Gyro bias & $0.01[\mathrm{deg} / \mathrm{sec}]$ \\
\hline Initial longitude angle & $30.6[\mathrm{deg}]$ & Shut-off time & $59.34[\mathrm{sec}]$ \\
\hline Initial velocity & $0.00[\mathrm{~m} / \mathrm{s}]$ & Azimuth misalignment & $3[\mathrm{arc} \mathrm{min}]$ \\
\hline Initial heading angle & $260.0[\mathrm{deg}]$ & Maximum angular rate & $2.5[\mathrm{r} / \mathrm{s}]$ \\
\hline Initial altitude & $175[\mathrm{~m}]$ & Sampling rate & $10[\mathrm{~m} \mathrm{sec}]$ \\
\hline
\end{tabular}

From the trajectory data, under the assumption of initial misalignment angles is chosen as 1 $\left[{ }^{0}\right]$, measuring error of velocity is $0.5[\mathrm{~m} / \mathrm{s}]$, and the turn maneuver with maximum variation of heading angle 10deg from plane of fire.

Table (3) shows the different convergence rates of $\psi_{\mathrm{x}}, \psi_{\mathrm{y}}$ and $\psi_{\mathrm{z}}$ for 10-states and 12-states in the cases of straight level flight, and turn level maneuver. Also, figures $(8,9)$, and figures $(10,11)$ shows the result of azimuth estimated error, for 10-states and 12-states in the above two cases and figure 12 shows some of the missile flight parameters. 
Table 3. Different convergence rates of misalignment angles

\begin{tabular}{||l|l|c|c|c|}
\hline & \multicolumn{1}{|c|}{ Case } & $\Delta \psi_{\mathrm{x}}[\mathrm{sec}]$ & $\Delta \psi_{\mathrm{y}}[\mathrm{sec}]$ & $\Delta \boldsymbol{\psi}_{\mathrm{z}}[\mathrm{sec}]$ \\
\hline \multirow{2}{*}{ 10-states (2- channel) } & Straight level-flight & $\mathbf{- 6 . 3 7 3 3}$ & $\mathbf{- 2 9 . 1 4 1 5}$ & $\mathbf{- 2 1 1 . 3 6 9 9}$ \\
\cline { 2 - 5 } & turn level maneuver & $\mathbf{0 . 8 4 3 0}$ & $\mathbf{- 0 . 5 8 9 8}$ & $\mathbf{- 1 . 7 3 8 5}$ \\
\hline 12-states (3- channel) & Straight level-flight & $\mathbf{- 6 . 4 9 8 2}$ & $\mathbf{- 2 8 . 8 4 1 6}$ & $\mathbf{- 2 3 1 . 7 7 3 7}$ \\
\cline { 2 - 5 } & turn level maneuver & $\mathbf{- 0 . 2 7 5 7}$ & $\mathbf{0 . 3 0 2 2}$ & $\mathbf{- 3 . 7 1 5 9}$ \\
\hline
\end{tabular}

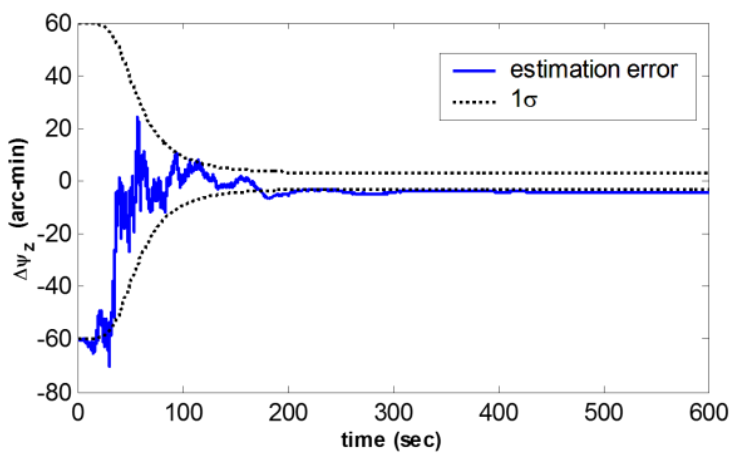

Fig. 6 Azimuth error estimate with straight level in 10 states

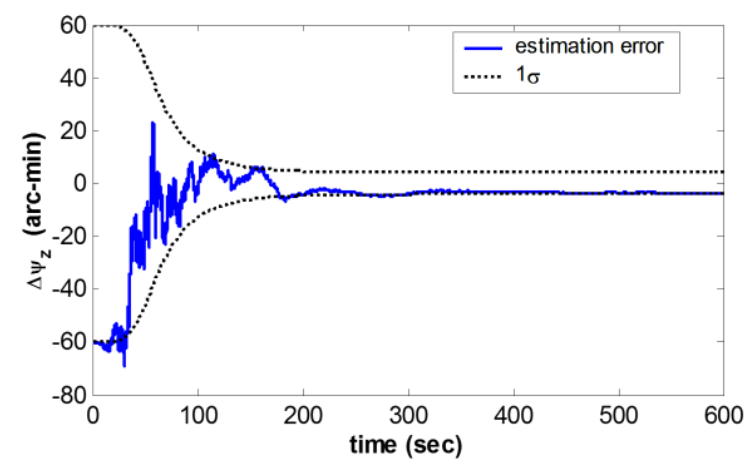

Fig.8 Azimuth error estimate with straight level in 12 states

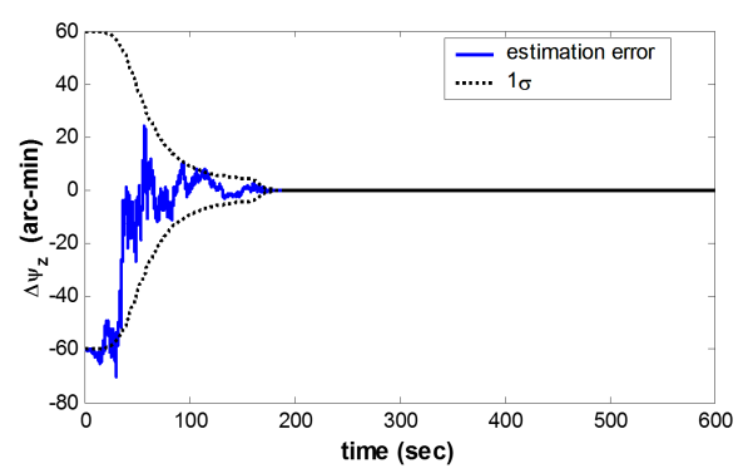

Fig. 7. Azimuth error estimate with turn level in 10 states

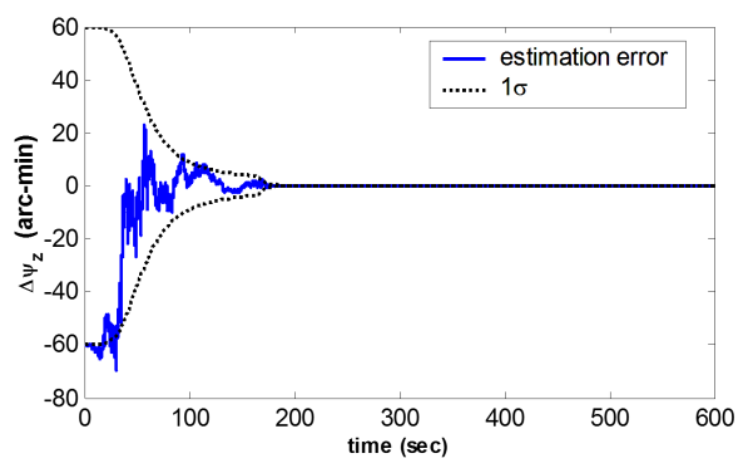

Fig. 9 Azimuth error estimate with turn level in 12 states

\section{Conclusions}

This paper presents a novel alignment method based on wander frame for SINS (strapdown inertial navigation system) whose initial attitude information is completely unknown. During the course mode, the cosine and sine of the wander angle is defined as the filter states, and modeled as the velocity/position error in the geographic frame. Azimuth error state in the fine mode of the filter is defined as wander angle error to provide continuous estimation of navigation states. The attention was focused on the derivation and programming of NMSW algorithm for SINS wanders azimuth mechanization. Based on the trajectory simulator data from the missile equation of motions the process of simulation and computation is highlighted. Also, we introduced the error model, which appropriately describes the INS behavior during IFA. We then substantiated its adequacy for consideration as a piece-wise constant system (PWCS). Using PWCS we can simplify in-flight alignment Observability analysis. The simulation results showed the effect of the variation of initial large azimuth misalignment angle for two-channel and three channels including the un-damped channel by using the extended Kalman filter (EKF). 

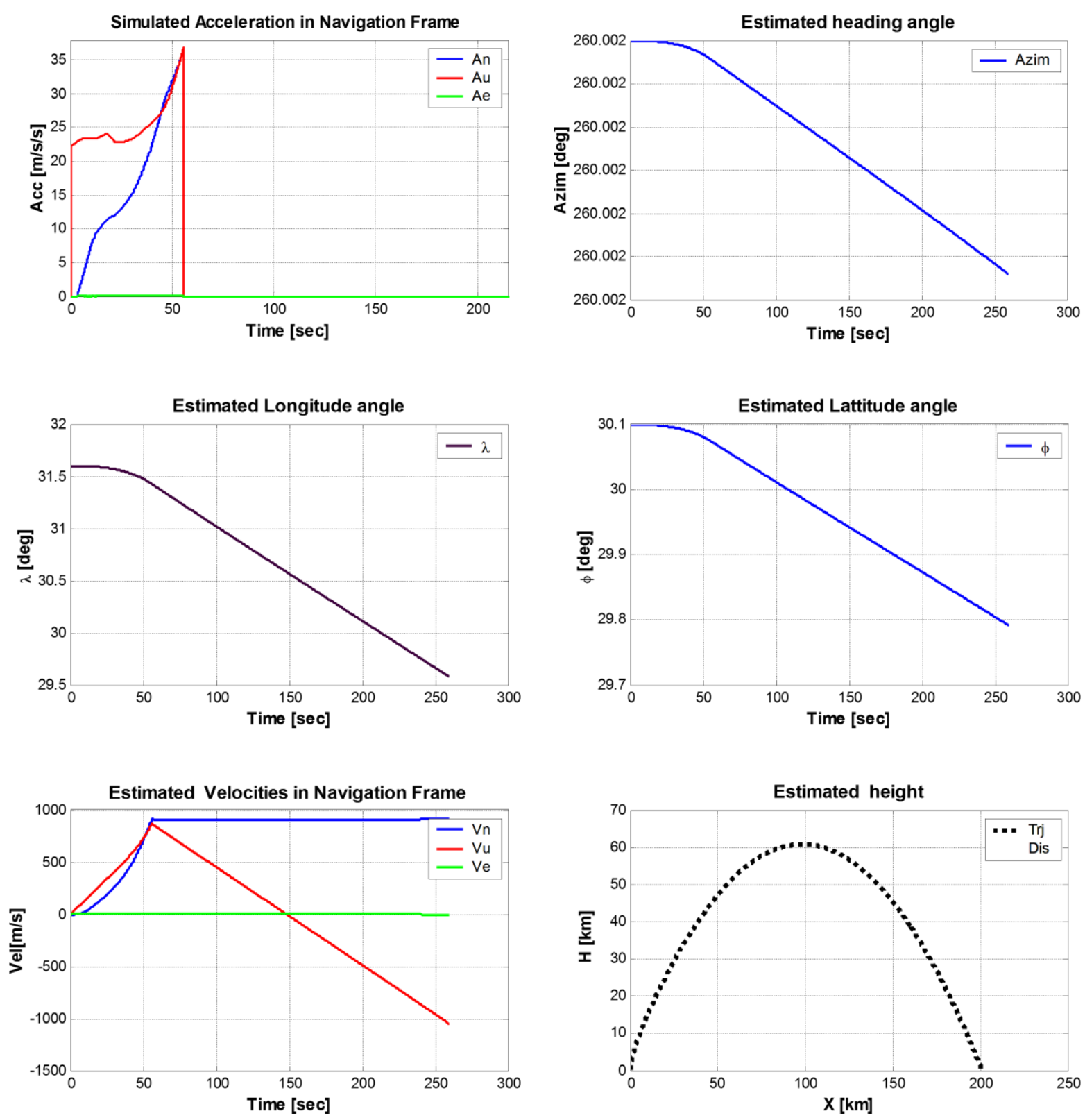

Fig.10 Estimated flight parameters of the missile

\section{References}

[1] Jong-Hyuk Kim, Stuart Wishart and Salah Sukkarieh, 'Real-time Navigation, Guidance, and Control of a UAV using Low-cost Sensors", Australian Centre for Field Robotics University of Sydney, NSW 2006, Australia.

[2] M. A. Elleithy, M. O. Tantawy, M. E. Ghoniemy, "Development Of Six-Degrees of Freedom Model For Surface to Surface Strap down Ballistic Missile" GC-5, Third ASAT Conferences M.T.C. Cairo, Egypt 1989.

[3] George M. Siouris, "Aerospace Avionics Systems "A Modern Synthesis Air Force Institute of Technology 1993.

[4] El-sheikh, G. A.,"An Investigation into the performance of strap down Inertial Navigation Systems" Thesis, M.T.C., Cairo, Egypt 1990. 
[5] Ibrahim I. Arafa, M. I. Latif, Zhong .H.Yue " Correction of the Flight Path Based on Error Estimation Of SINS Ground Alignment Using Modern Computer Techniques" Beihang University, Beijing 100083, Conferences second Asian workshop of foundations of software. Nanjing China DEC. 2003.

[6] Ibrahim Ismail Arafa,' Analysis and Design of Guidance Navigation and Control Systems for Missile Applications', Beihang University Sep. 2004

[7] Rodeny E. Stubbs, Whittak H. Huang and Eric Schimtz "Integration of GPS into Ballistic Navigation Solution" Lockheed Martin Astronautics By American Institute of Aeronautics and Astronautics, Inc. 1997

[8] M. Napier, "Integration of Satellite and Inertial Positioning Systems" Institute of Engineering Surveying and Space Geodesy, University of Nottingham, Vol. 43, 1995

[9] Ne Eduardo, Durrant-Whyte Hugh. Department of Mechanical and Mechatronic Engineering University of Sydney, 2006, NSW, Australia, 0-8186- 8025-3/97IEEE 1997

[10] Ye Fuh Jiang Chung Shan Institute of Science and technology, Taiwan "Error Analysis of Analytic Coarse Alignment methods" IEEE Transaction on Aerospace and Electronic, 2. May 1997.

[11] Kayton, M., and Fried, W.R.(1969) "Avionics Navigation Systems"

[12] Huddle, J. R. (1983) "Inertial navigation system error-modelconsiderations in Kalman filtering applications". In C.T. Leondes (Ed.),Control and dynamics Systems. New York: Academic Press, 20 (1983)

[13] Goshen-Meskin, Bar-Itzback Israel Aircraft and Institute of Technology "Observability Analysis of Piece-Wise Constant Systems-Part II: Application to inertial Navigation InFlight Alignment" IEEE Transactions on aerospace and electronic systems Vol. 28, No. 4, October, 1992.

[14] Goshen-Meskin, Bar-Itzback Israel Aircraft and Institute of Technology "Observability Analysis of Piece-Wise Constant Systems with Application to inertial Navigation" IEEE Transactions on aerospace and electronic systems Vol. 2, December 1990.

[15] Mostafaelzoghoby M.S. Thesis "Error Estimation of INS Alignment on Stationary and Moving Bases through Observability Analysis” M.T.C. JULE 2011. Cairo, Egypt. 